\title{
Parametric Study of Variable Renewable Energy Integration in Europe: Advantages and Costs of Transmission Grid Extensions
}

\author{
Katrin Schaber ${ }^{\mathrm{a}, \mathrm{b}, *}$, Florian Steinke ${ }^{\mathrm{c}}$, Pascal Mühlich ${ }^{\mathrm{b}}$, Thomas Hamacher ${ }^{\mathrm{a}}$ \\ ${ }^{a}$ Institut für Energiewirtschaft und Anwendungstechnik, Technical University Munich, \\ Arcisstr. 21, 80333 Munich, Germany \\ ${ }^{b}$ Max-Planck-Insitute for Plasmaphysics, Boltzmannstr. 2, 85748 Garching, Germany \\ ${ }^{c}$ Siemens Corporate Technology, Munich, Germany
}

\begin{abstract}
Wind and solar energy will play an important role in the decarbonization of the European electricity generation. However, high shares of these variable renewable energies (VREs) challenge the power system considerably due to their temporal fluctuations and geographical dispersion.

In this paper, we systematically analyze transmission grid extensions as an integration measure for VREs in Europe. We show the effects of grid extensions for fundamental properties of the power system as a function of the penetration penetration and mix of wind and solar energy. Backup capacity requirements and overproduction are reduced with a powerful overlay transmission grid. We determine the costs of the grid extensions in dependence of the VRE penetration and mix and find, that the grid integration costs remain below $25 \%$ of the VRE investment costs for all concievable VRE configurations. Furthermore, robust design features of future power systems in
\end{abstract}

*k.schaber@tum.de 
terms of grid geometry and flexibility requirements for backup technologies are identified.

We apply a spatially and temporally highly resolved techno-economic model of the European power system for our analysis.

Keywords: Variable Renewable Energies, grid integration costs, ideal mix

\section{Introduction}

2 Large shares of renewable electricity generation are a promising possibility

3 to address global warming and the rising scarcity of hydrocarbon fuels (IPCC,

4 2011; McKinsey et al., 2010; PWC et al., 2010). In Europe, an important

5 part of the renewable generation will come from wind and solar PV energy

6 due to the observed learning and growth rates and the political support 7 schemes (Edenhofer et al., 2010; IEA, 2011b). Furthermore, the technical

8 potential of wind and solar energy is largely sufficient to supply the European

9 electricity demand (Tzscheutschler, 2005; Brückl, 2005; Hoogwijk, 2004).

10 However, these two resources are geographically dispersed and underlie

11 strong temporal fluctuations. These characteristics of solar and wind en-

12 ergy - for which we term them variable renewable energies (VREs) - make

13 their integration into the existing power supply system difficult. Today's

14 electricity system is based on centralized supply in proximity to load cen-

15 ters. The different power plant types are designed to follow the hourly load.

16 International transmission of electricity and storage only play a minor role.

17 This changes significantly in energy systems with high shares of renewable

18 energy and entails major integration challenges for the power system, such

19 as the connection of remote sites of high VRE potential, the low reliability 
of VRE, occuring overproduction etc. (IPCC, 2011). Therefore, a power supply system with large VRE penetration, should be designed in a way that integration challenges are kept minimal. Renewable energy policies should be based on solid knowledge about these challenges and which system design features can be advantageous. Policy makers may face questions about the implications of a given VRE penetration level to the power system. How to design cost-effective renewable supply? Which mix between wind and solar energy has to be chosen to keep overproduction minimal? How can the reliability of VRE be increased, i.e., the required backup 1 capacity reduced? What benefits arise from grid extensions?

In this paper, we provide an overview of the system implication of wind and solar PV energy and investigate a way to partly overcome these: transmission grid extensions. We compare the integration challenge of a large range of VRE scenarios in Europe and investigate the role of transmission grid extensions as a measure to address the temporal fluctuations and the geographical dispersion of VRE. We systematically analyze the effect of different VRE penetration levels and mixes between wind and solar power on fundamental power system properties, such as overproduction and required backup capacity, as function of grid extensions. This allows us to identify benefits of transmission grid extensions for VRE integration. We quantify the costs of a powerful overlay transmission grid for VRE integration for different scenarios. These costs are called "grid integration costs", i.e., the additional costs per installed MW capacity due to grid extensions for VRE integration.

\footnotetext{
${ }^{1}$ In this study, we define "backup" power plant, as all dispatch-able power plants in the electricity sector, i.e., all capacity except VRE technologies.
} 
Finally, robust design features of a power system with high VRE penetration are presented: We identify crucial grid connections in Europe and study the role and suitability of different backup technologies.

Already in the early 1970s, grid extensions have been identified as a necessary condition to achieve large shares of renewables: Buckminster Fuller (1971) proposes a global link to harvest energy resources most efficiently. On the European level, first detailed studies on the feasibility and principal advantages of a "super-grid", i.e., a powerful transmission grid, have been presented by Biberacher (2004); Czisch (2005) and DLR (2006). In the light of actual growth of wind and solar energy and the institutional and political barriers to grid extensions, analyses of the actual and near future situation have been carried out recently. In TradeWind (2009), overall grid extensions for wind energy until 2030 are quantified. Grid strengthening can furthermore reduce the impacts of VRE on the electricity market and their participants, 57 as shown by Schaber et al. (2011). On a longer time horizon, McKinsey et al. (2010) and PWC et al. (2010) present possible roadmaps towards a carbon free power supply in 2050 in Europe. McKinsey et al. (2010) present a technically and economically feasible pathway to a zero-emitting European power sector in 2050, which includes nuclear power, carbon capture and storage, 62 and renewable energies. PWC et al. (2010) propose a $100 \%$ renewable sce63 nario for Europe under the prerequisiste of a European "SuperSmartGrid". ${ }_{64}$ Furthermore, the amount of necessary backup capacity can be reduced sub${ }_{5}$ stantially through grid extensions, as Aboumahboub et al. (2009) show for 66 the European and the global case.

${ }_{67}$ In addition to these detailed techno-economic studies mostly based on 
advanced power system modeling, important information can be drawn from statistical analyses of the supply time series. Giebel (2000) and Greenpeace and 3E (2008) quantify the statistical smoothing of wind volatility through increased interconnection between generating regions. In Grotz (2009) and Heide et al. (2010) the seasonal fluctuations of wind and solar availability in Europe are understood as chance rather than challenge: The optimal combination of wind energy, mainly available in winter, and solar energy, mainly available in summer, allows to minimize the need for inter-seasonal storage or backup power. While analyses based on energy models mostly are based on a limited number of scenarios, statistical studies cover a larger range of the possible system configurations.

In this study, we apply a complex model to a wide parameter space of possible system configurations for different VREs penetration levels and mixtures. This allows to study the effects of VRE on a power system. For each system configuration, we determine necessary transmission grid extensions and analyse their effects on VRE integration. We combine highly resolved eight-year meteorological time series with a detailed power system model, which makes our approach very robust.

Storage capacities are assumed to remain at today's level, as the focus of this study lies on grid extension as a measure to integrate VREs. Storage can provide important contributions to integrate renewable energies and a variety of application fields for the different technologies exist (Kuhn and Kühne, 2011). However, today, only few technologies are costcompetitive (Pieper and Rubel, 2011). Another simplification in our approach is, that we only model the high-voltage transmission grid. The un- 
derlying distribution grid is not included in our analysis.

Our paper proceeds as follows: the methodology is described in Section 2. Results are presented in Section 3: we analyze the technical and economic feasibility and suitability of different VRE supply configurations with and without grid extensions (Subsection 3.1). The costs and structure of the European high voltage transmission grid, necessary to integrate VREs, are quantified in Subsection 3.2. The suitability of different backup technologies is studied in Subsection 3.3. An illustration of the results is provided in a casestudy in Subsection 3.4. Finally, we discuss our results and conclude in Section 4

\section{Methodology}

\subsection{Definition of parameter space}

To carry out a systematic analysis of VRE integration, we introduce a two dimensional parameter space, defined by the total share of VREs in the satisfaction of load $\alpha$ and the mix between solar and wind energy $\beta$.

$$
\begin{aligned}
\alpha & =\frac{\sum_{t} \min \left(E_{W I N D}(t)+E_{S U N}(t), D(t)\right)}{\sum_{t} D(t)} \\
\beta & =\frac{\sum_{t} E_{W I N D}(t)}{\sum_{t}\left[E_{W I N D}(t)+E_{S U N}(t)\right]}
\end{aligned}
$$

where $E_{W I N D}(t)$ is the total hourly energy generation from wind at time $t$, $E_{S U N}(t)$ the generation from solar photovoltaics, and $D(t)$ the demand. Note that oversupply, i.e., renewable generation that exceeds the demand at time $t$, is disregarded in the definition of $\alpha$. As we assume current and therewith limited storage possibilities, most excess generation has to be discarded. 
Traditionally, power supply systems are built to follow the load and this determines the power generation, grid and storage infrastructure. However, in highly renewable scenarios the system is mostly determined by the share and type of the VREs. VREs determine the residual load, which has to be satisfied by the power supply system. The two parameters $\alpha$ and $\beta$ are thus central in the comparison of systems.

\subsection{Model structure and assumptions}

To determine the behavior of the European power supply system for each $\alpha$ and $\beta$, we employ the European power system model URBS-EU based on linear optimization of total system costs (Schaber et al., 2011). This regionally and temporally highly resolved model of the European power supply system computes the cost-optimal production schedules for the dispatchable (backup) power plants and a cost-optimal electricity transport across Europe. On request, it can also optimize the infrastructure, i.e., the power plant fleet or the interregional electricity transmission capacities. Important properties of the electricity supply system, such as the satisfaction of demand in each model region and time step or the transmission, storage and conversion losses are included in the optimization via boundary conditions.

In the parametric study, VRE capacities are determined by the requirements to achieve certain shares $\alpha$ and $\beta$, while the residual power system is subject to the cost-optimization implemented in the power system model URBS-EU. Total system costs include VRE, backup and storage capacity, operation, maintenance and fuel and carbon costs and the electricity transmission grid. The power system model has hourly resolution and divides Europe geographically into 83 model regions (see Figure 1). Power plants are aggregated with 


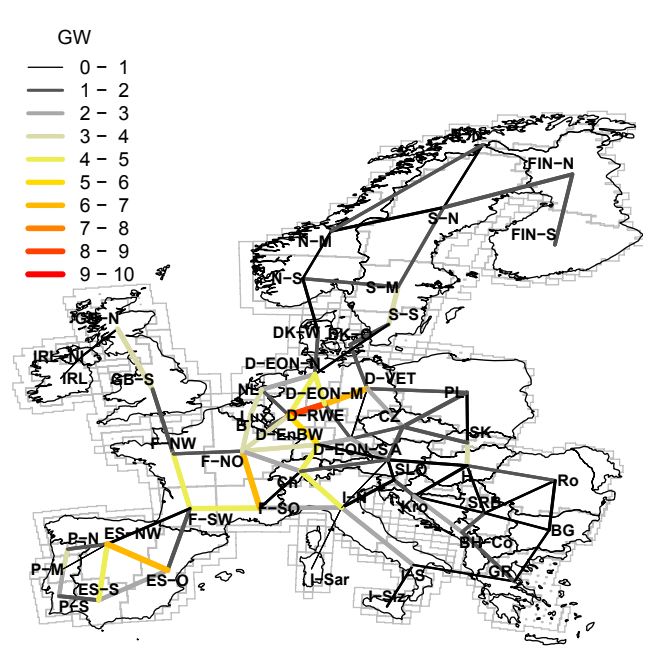

Figure 1: European model regions and capacity of aggregated ENTSO-E transmission capacities.

respect to type and region. The optimization problem is kept linear to allow feasible large scale modeling.

The hourly availability of VREs is taken into account through the capacity factor $s_{i}(x, t) \in[0,1]$, which is computed from an eight-year time series (2000-2007) of meteorological data for each region $x$ and VRE type $i \in\{W I N D, S U N\}$ (Heide et al., 2010). $E_{i, \alpha, \beta}(x, t)$, the hourly power production per region and VRE type is the product of $C_{i, \alpha, \beta}(x)$, the capacity per model region and type and the time dependent capacity factor $c f_{i}(x, t)$ for each VRE technology. The total energy production from VREs at time $t$ is given by $E_{i, \alpha, \beta}(t)=\sum_{x} E_{i, \alpha, \beta}(x, t)$.

We assume that the distribution of wind and solar capacities $C_{i, \alpha, \beta}(x)$ is proportional to the potential of wind and solar power production, respec- 
tively, i.e., to the Full Load Hours (FLHs, shown in Figure 2). This distribution of capacities reflects that the costs of electricity from renewable energies is cheapest on sites with the largest potential. This is not happening today, since different political support schemes and targets have lead to strongly differing installation rates in various countries (IEA, 2011a). However, from a European perspective, the most cost-effective way to harvest renewables in the long term perpective, is to build the largest capacities where the potential is highest. As this study departs from a European point of view, we assume, that more VRE capacities will be built in regions with higher FLHs. The contribution of wind offshore capacity to the total European wind capacity is assumed to be $50 \%$.

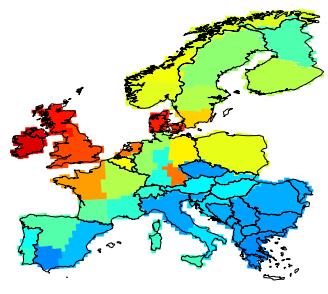

(a) Wind Onshore

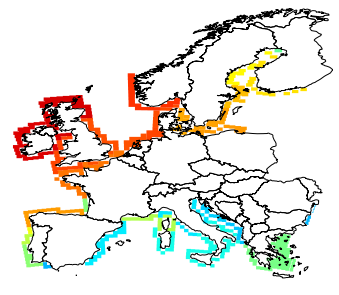

(b) Wind Offshore

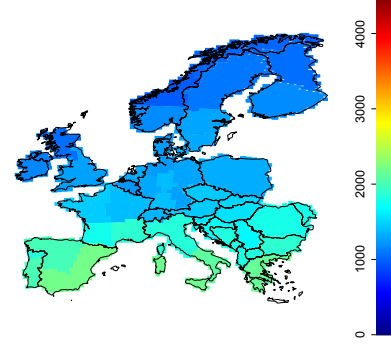

(c) Solar Photovoltaics

Figure 2: Full Load Hours of wind and solar PV supply

Industrial and residential demand for electricity is assumed to remain at today's level in the entire study. While population and economic growth raise electricity demand, efficiency measures counteract (McKinsey et al., 2010). To account for these diametrical trends and to ensure comparability, we use current hourly load profiles and current total electricity demand.

Today's power system infrastructure is included in the model. The ac- 
tual generation and storage capacities per model region are determined from a Geo-referenced power plant database (Schaber et al., 2011). As we focus on the role of grid extensions only, additional storage capacity is not allowed for. Today's transmission grid is obtained from freely available data on the European high voltage electricity grid (ENTSO-E, 2010b, 220kV and $380 \mathrm{kV}$ ). The capacity of the transmission lines is computed based on their natural load, i.e., the power level where no reactive losses occur and is benchmarked with cross boarder net transfer capacities published by ENTSO-E (see Figure 1 ENTSO-E, 2011b). The costs and technical parameters are based on scientific studies (IEA, 2010a; McKinsey et al., 2010, see Table A.3). The hourly load curves for the years 2000-2007 stem from the European Transmission System Operator (ENTSO-E, 2010a). To model the power exchange along the transmission lines we perform a simplified simulation of electricity transport between regions. Kirchhoff's first law, the conservation of currents in each node of an electricity network, is respected in our model, while the second, the voltage law is not modeled. This results in a transport model omitting load flow. Due to computational restrictions and low data availability, the distribution network is not included.

To compare possible VRE supply systems for a wide range of the parameters $\alpha$ and $\beta$ we need to run the model many times. Yet, the computations with the fully-detailed model are time- and memory-consuming and are practically limited to about 6 typical weeks for each $\alpha$ and $\beta$ value. We therefore developed a simplified version of the model where the technical details of the backup power plants are simulated in less detail. This allows to compute good approximations of important power system quantities listed in equation 3 to 
6. using all eight years of available VRE generation time-series (Subsection 3.1). The full model version is applied for the computation of grid extensions and backup dispatch (Subsection 3.2 to 3.3). Six representative weeks from meteorological data of 2007 are used. We performed a sensitivity analyses and the results show that the results in terms of grid extensions and backup mix do not change significantly if longer timeseries or another meteorological year are employed.

Fundamental power system properties are the necessary VRE and backup capacities, $C_{V R E}$ and $C_{B}$, the amount of overproduction $O P$, and the distance between supply and demand time curve $\Delta$. If the "misfit parameter" $\Delta$ was zero, there would be no VRE integration challenge.

$$
\begin{aligned}
C_{V R E}(\alpha, \beta) & =\sum_{x, i} C_{i, \alpha, \beta}(x) \\
C_{B}(\alpha, \beta) & =\max _{t}\left(D(t)-E_{V R E, \alpha, \beta}(t)\right) \\
O P(\alpha, \beta) & =\frac{\sum_{t} \max \left(E_{V R E, \alpha, \beta}(t)-D(t), 0\right)}{\sum_{t} D(t)} \\
\Delta(\alpha, \beta) & =\sum_{t}\left|D(t)-E_{V R E, \alpha, \beta}(t)\right|
\end{aligned}
$$

Here, $D(t)$ is the sum of the regional demands $D(x, t), E_{V R E, \alpha, \beta}(x, t)=$ $\sum_{i} E_{i, \alpha, \beta}(x, t)$, and $E_{V R E, \alpha, \beta}(t)=\sum_{x} E_{V R E, \alpha, \beta}(x, t)$.

\section{Results}

\subsection{Effects of grid extension on VRE integration challenges}

To study VRE integration, we compute the above described power system properties for a case with optimal grid extensions and one without. In the first case, we assume unrestricted electricity transport across the entirety 
of Europe, in the second case, each model region acts independently. This allows us to assess the difficulty of VRE integration and the effect of grid extensions.

To aggregate the results in the no-grid case, the regional results are summed up in accordance to $\alpha$ and $\beta$. In this aggregation we assume that the mix between wind and solar power $(\beta)$ is identical in all regions across Europe. Figure 3 shows the necessary VRE capacity to realise each $\alpha-\beta$ combination as well as the required backup capacity, overproduction and the misfit between supply and demand $\Delta$ (see equation 6). In the left column, an optimal grid within Europe is assumed, in the right column, no interconnection is assumed.

We present our results through color coding in the parameter space. The penetration level of VRE $\alpha$ and the share of wind $\beta$ span the parameter surface, where $\alpha$ is plottet on the abscissa and $\beta$ on the ordinate (see Figure 3). The value of a variable is represented through color levels. Some combinations of $\alpha$ and $\beta$ are infeasible. For these points on the surface, no variable value exists and the surface remains white: Large VRE shares with high solar contribution are infeasible. With grid extensions, the maximal possible share amounts about $\alpha=50 \%$, if only solar PV is used ( $\beta=0$, left column of Figure (3). As the sun only shines during day time and we assume no storage extensions, it can be easily understood, that $100 \%$ satisfaction of demand is impossible with solar energy only. Without grid extensions larger areas of the parameter space remain empty and thus infeasible (right column of Figure 3 ). 
The first row of Figure 3 shows that VRE capacity rises steeply with increasing $\alpha$ in the parameter space. The realization of a configuration at the rim of the colored area in the parameter space translates into a VRE each VRE technology - solar PV, wind Onshore and wind Offshore is limited to five times the peak load. The theoretical maximal VRE capacity thus amounts to 15 times the peak load. As for very high shares of VREs mainly wind capacity is needed, the total VRE capacity is 10 times the peak load. If larger overcapacity was allowed, higher shares of VRE were possible, if lower overcapacity is allowed, lower VRE shares can be achieved. The dotted line in Figure 3 shows the maximal possible shares with a capacity limit of 20 times the peak load for each of the three technologies, the dashed line shows the maximal shares with a limit of two times the peak load. The first case corresponds to a 59 fold overcapacity and the second case limits the possible parameter space considerably. Therefore we chose an intermediate level of VRE capacity limit of five times the peakload per VRE technology. 

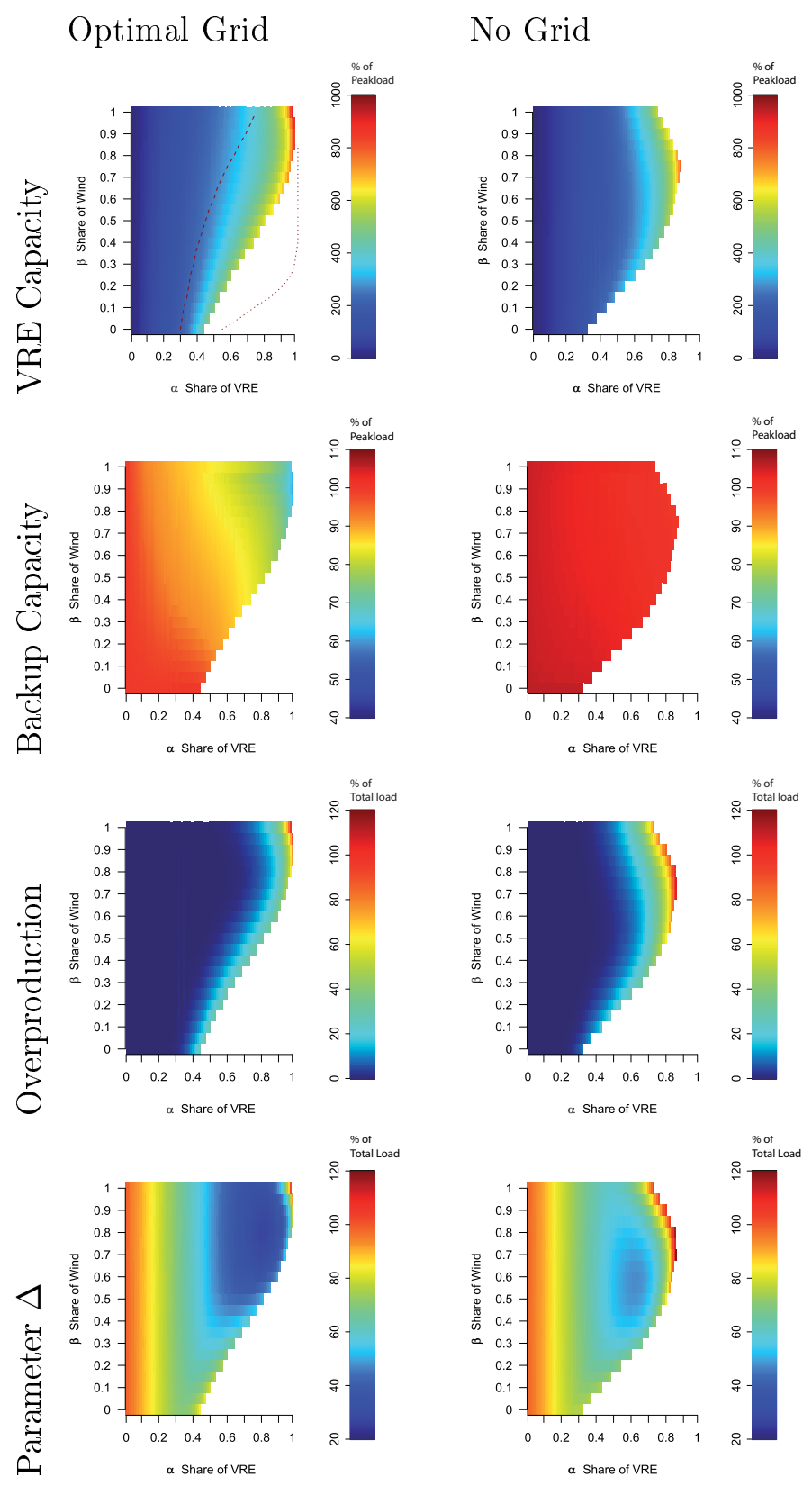

Figure 3: Fundamental properties of VRE power systems in Europe with optimal grid and without grid. The first row indicates the necessary VRE capacities for each $\alpha$ and $\beta$. The three lower rows provide measures for the integration challenge of these VRE capacities. In the results shown here, the capacity of each VRE technology is limited to five times the peak load. The lines in the upper left plot indicate the maximal possible VRE shares with lower or higher VRE capacity limits: a limit of two times peak load per VRE technology results in the dashed lines, a limit of 20 in the dotted line. 
To ensure reliable electricity supply, sufficient backup capacity is necessary. The second row of Figure 3 shows that all VRE configurations need considerable backup capacities. This is due to the fluctuating availability of VREs and the resultung low reliability of these energy sources. With an an optimal grid necessary backup can be reduced to $60 \%$ of the peak load. Without grid the total backup remains close to $100 \%$ of the peak load in the entire parameter space. For both cases, the maximum of the European load serves as reference. As the peak load does not happen at the same time all over Europe, the sum of local peak loads exceeds the European peak load. This is why the backup capacity requirements can reach values larger than $100 \%$. The backup capacity is determined using the simplified model version, which does not include transport losses, ramp up or availability restrictions. The presented capacity requirements should thus be understood as lower limits, which increase if all technical details and security margins are taken into account. The current power generation capacity in Europe, for instance, amounts about 1.4 times the peak load (ENTSO-E, 2010b).

The VRE overcapacity leads to overproduction, shown in the third row of Figure 3. The overproduction's dependence on $\alpha$ is similar to an exponential function: it is very flat in proximity to the origin, but from a certain VRE share on, it rises steeply. The detaching point varies with the share of wind energy: more wind energy leads to less overproduction. Also, higher levels of linkage entail lower excess production for identical VRE shares. The optimal mix between wind and solar power to achieve minimal overproduction for a given VRE share depends on the level of interconnection: With an optimal grid, a mix of $85 \%$ wind to $15 \%$ solar energy supply is ideal, while in the 
non-connected case, lower shares of wind (75\%) are favorable. Wind energy is highly variable, while solar energy supply is less stochastic and closer to the load pattern in the temporal and spatial domain. Solar capacity is furthermore more evenly distributed than wind (see Figure 2). Therefore, wind energy profits more from interconnection, while solar energy has a systematic advantage in low-connection cases.

If one considers storage as an integration option for VRE, minimal misfit $\Delta$ (equation 6) would be desirable, as this translates into minimal energy to be stored and released in total. With an optimal grid, a VRE share of $82 \%$ and a wind contribution of $80 \%$ result in minimal $\Delta$, i.e., minimal adaption needs of the residual power system. Again the lower interconnection cases favor higher shares of solar energy. However, the absolute misfit is lower with an ideal grid, i.e., the misfit between VRE supply and demand can be reduced through grid extensions. The grid smoothens the wind supply and the resulting total European wind generation pattern is closer to demand than the solar pattern. Solar generation correlates well with demand on a diurnal timescale, but can not provide a basis to cover electricity demand at night. Without grid extensions, wind supply is not smooth enough and thus does not fit very well to the demand.

Based on the VRE and backup capacities, average European Costs of Electricity (CoE) can be determined. Assuming $40 \%$ capacity margin as of today, and coal and gas combined cycle power plants as backup technologies, the CoE range between 80 and $170 € /$ MWh with grid extension and can reach more than $207 € / M W h$ without grid extensions (see Table 1). Compared to current CoE of conventional technologies, such as $55 € / \mathrm{MWh}$ for 


\begin{tabular}{l||l|l}
$\ell$ & high costs & low costs \\
\hline \hline & \multicolumn{2}{|||}{ Optimal Grid } \\
\hline$\alpha=10 \%, \beta=50 \%$ & 88 & 76 \\
$\alpha=50 \%, \beta=70 \%$ & 131 & 79 \\
$\alpha=60 \%, \beta=60 \%$ & 140 & 80 \\
$\alpha=80 \%, \beta=90 \%$ & 170 & 87 \\
\hline & \multicolumn{3}{c}{ No Grid } \\
\hline$\alpha=10 \%, \beta=50 \%$ & 90 & 83 \\
$\alpha=60 \%, \beta=80 \%$ & 143 & 90 \\
$\alpha=80 \%, \beta=90 \%$ & 207 & 131
\end{tabular}

Table 1: Average European Costs of Electricity (CoE) per MWh load for different VRE configuration and different levels of interconnection. Two cost scenarios for VREs are studied. In the high costs case, VRE investments costs are assumed at $2500 € / \mathrm{kW}$ for Solar PV, $1800 € / \mathrm{kW}$ for Wind Onshore and $3370 € / \mathrm{kW}$ for Wind Offshore; low costs are 800,1000 and $1300 € / \mathrm{kW}$ respectively (see Table A.3). 
coal power plants and $110 € / \mathrm{MWh}$ for gas turbines (with a carbon price of $20 € /$ t, see Table A.3), high VRE scenarios might not be economically viable. With an increased carbon price of $100 € / t$, VRE scenarios score better in the comparison, as the CoE of coal power plants rises to $115 € / \mathrm{MWh}$ and the one of gas turbines to $155 € /$ MWh. The costs vary considerably with VRE share and mix, but also with the assumed wind and solar investment costs and we therefore present two costs scenarios in Table 1. For high $\alpha$, large VRE overcapacities are necesary and therefore the CoE, computed with respect to electricity demand, exceed the long run marginal costs of the VRE technologies themselves. Higher costs in the non-connected case are caused by larger backup and VRE capacity needs. The CoE exclude costs for grid extension, treated separately in Subsection 3.2 .

The comparison of adaption needs of the power system and costs for highly renewable supply with and without grid extension shows that increased interconnection bears important benefits for VRE integration. Grid extension reduces VRE and backup capacity needs and with it the costs. Furthermore, smaller misfit between supply and demand plus lower overproduction occur.

\subsection{Grid costs and structure}

The foregoing section showed, that some impacts of VRE to the power system can be reduced through a powerful overlay transmission grid across Europe. The costs and dimensions of this grid are specified in this section. 

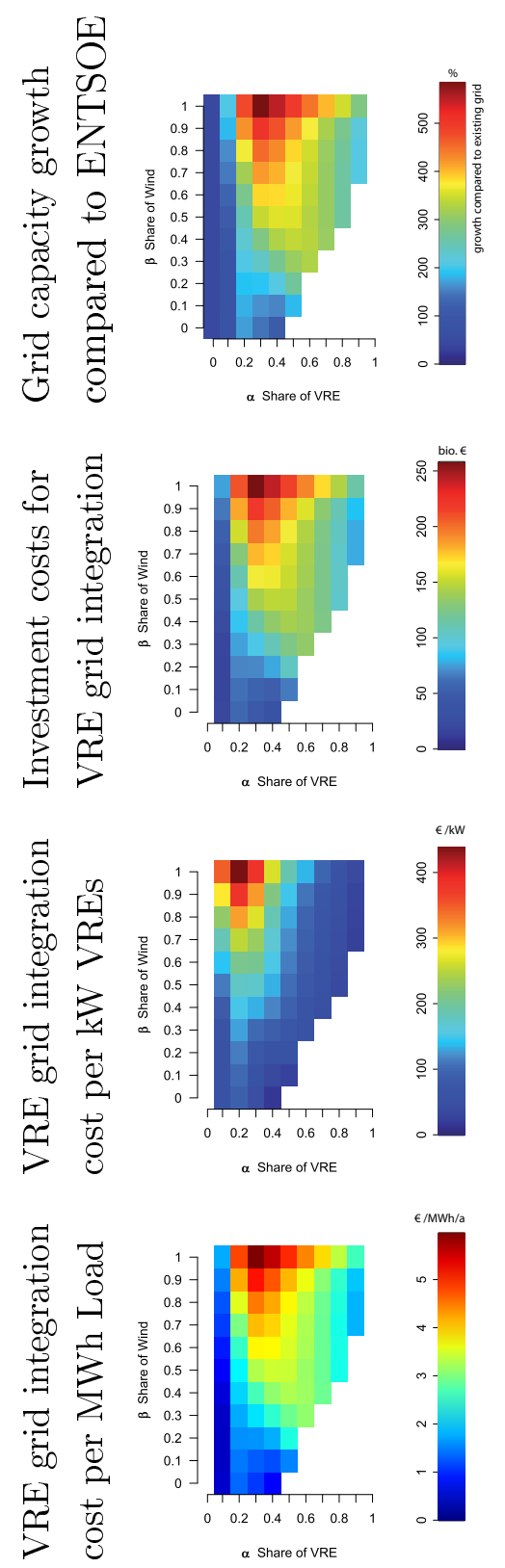

Figure 4: Grid extensions and costs. Grid extensions are the increase in grid capacity and length (MWkm) with respect to the current ENTSO-E grid. Costs are the additional costs due to VRE deployment. They are deduced from incremental grid extensions between the reference case where $\alpha=\beta=0$ and configurations with $\operatorname{VRE}$ contribution $(\alpha>0)$. 
Figure 4 shows the total high voltage transmission grid capacity and its costs resulting from the optimization model URBS-EU. With increasing VRE share, the total high voltage transmission grid capacity increases to up to the six fold capacity of today's existing grid. The capacity of the grid is measured in GWkm and thus contains its length and capacity. For medium VRE shares of $30 \%$ to $50 \%$, an overlay grid is very cost-effective, while in the cases with very high VRE shares the regional energy production already is high enough and the advantage of electricity transmission is lower. To assure reliable electricity supply, sufficient backup capacity has to be built (see Figure 3). We assume that the distribution of backup capacities in Europe is proportional to the load in each country, i.e., similar to today's situation. Each country has the necessary backup capacities available.In cases with very high VRE shares, these national backup capacities are used to satisfy the demand in the few hours of regional VRE shortage instead of grid extension for electricity import. Furthermore, grid capacity rises with $\beta$ : the linkage between regions is less prefereable for solar than for wind energy. This is due to the long correlation length of solar supply in Europe concerning intradiurnal variations (Grotz, 2009): it is night at about the same time all over Europe. Also, as pointed out earlier, solar supply is geographically more evenly dispersed in Europe and temporally closer to the load.

The total investment costs for grid extensions to integrate VRE reach 250 Billion $€$ (second row of Figure 4). Note, that the renewable grid integration costs are deduced from incremental grid extension between the reference case where $\alpha=\beta=0$ and configuration with VRE contribution $(\alpha>0)$. In the reference case, transmission grid extensions are cost-optimal as well. These 
grid extensions, due to a non-cost-optimal structure of the grid today are not due to VRE capacity additions and are substracted to obtain the investment costs for VRE integration. The grid investment costs correspond to less than $20 \%$ of the investment costs for VRE capacities for the high costs case presented in Table 1. Assuming low costs for VRE capacities, grid integration costs can reach up to $44 \%$ in extreme scenarios with $\alpha=20 \%$ and $\beta=100 \%$. In cases with wind contribution $\beta$ of $70 \%$ or lower, the maximal grid integration costs amount to $25 \%$ of the VRE investment costs. The renewable grid integration costs per VRE capacity are shown in the third row of Figure 4. In extreme cases, they reach $400 € / \mathrm{kW}$, but generally they range between 50 and $250 € / \mathrm{kW}$. The relative costs peak at medium VRE shares of $\alpha=20 \%$ to $40 \%$, which is due to the maximal total grid extensions at medium VRE shares and the large VRE capacities for high values of $\alpha$.

The specific renewable grid integration costs resulting form our analysis are in accordance with other studies. EWEA (2010) provides an overview of different studies, where the specific grid integration costs range between 10 and $370 € / \mathrm{kW}$ for scenarios of intermediate VRE share. McKinsey et al. (2010) find the grid integration costs to amount $83 € / \mathrm{kW}$ for the integration of $430 \mathrm{GW}$ wind and $816 \mathrm{GW}$ solar PV in Europe $(\alpha \approx 40 \%, \beta=30 \%)$. The IEA (2010b) estimates the investment costs for additional interconnection to be about $110 € / \mathrm{kW}$ for a scenario, which corresponds to $\alpha=40 \%$ and $\beta=80 \%$.

Sharing the annuity of investment costs for renewable grid integration between the consumers, additional $\mathrm{CoE}$ arise. They are shown in the last row of Figure 4 and reach at most $6 €$ per MWh load. We assume 40 years 
economic lifetime of the grid and a weighted average cost of capital of $7 \%$. Comparing the grid integration costs per MWh load to the CoE, our results show that they would increase by less than $5 \%$ due to the costs for grid extension (see Table 1 and Figure 4).

For the computation of VRE grid integration costs, we assume, that each country disposes of the necessary backup capacity. However, if national concerns about security of supply are disregarded and a cost-optimal distribution of backup capacties assumed, the amount of cost-optimal grid extensions increases. Figure 5 shows the total grid extensions for a case with cost-optimal backup capacity distribution.Here, grid extensions and backup capacities are optimized simultaneously in the model. This results in a cost-optimal distribution of backup capacities across Europe, which is largely driven by the grid geometry and VRE capacities. Backup capacity will in this case be built in well-connected regions to provide backup energy for all Europe across a powerful grid. Through international electricity transmission the total backup capacity is reduced and can be run more efficiently. It is cheaper to build more grid and reduce the costs for backup in turn, than to provide backup energy locally. Therefore, larger grid extensions result. From a system design perspective, this scenario would be very efficient, but politically it is highly unlikely, because countries would depend on its neighbours more than necessary. 


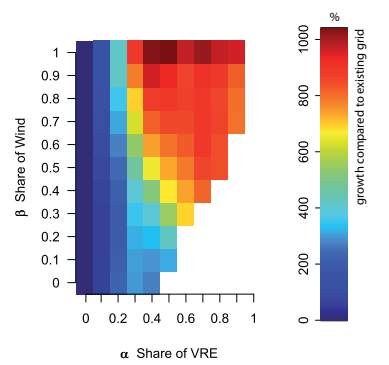

Figure 5: Grid extensions with cost-optimal backup capacity.

The geographical structure of the interregional transmission grid, which facilitates the VRE integration, is presented in Figure 6. It depicts the minimal grid extensions for any scenario with wind share between $60 \%$ and $100 \%$ for $\alpha=30 \%$ and $60 \%$. This "must have" grid shows the interconnections, which are cost optimal in any case. The structure of the grid is determined by the relative distribution of VRE capacities and load centers.Strong northsouth connections from north-western Europe to the load centers further north are prominent features. These power tramsission "highways" across Germany and France traversing the BeNeLux states and also across Poland export the wind energy generated in and around the North Sea southwards. Furthermore, an offshore grid in the North Sea as well as the connection of Spain and France across the Pyrenees, are crucial. With increasing VRE share, north-south connections in central Europe and the connection of Italy over the Alps to the rest of Europe gain importance. 
$\alpha=30 \%, \beta=60 \%$ to $100 \%$

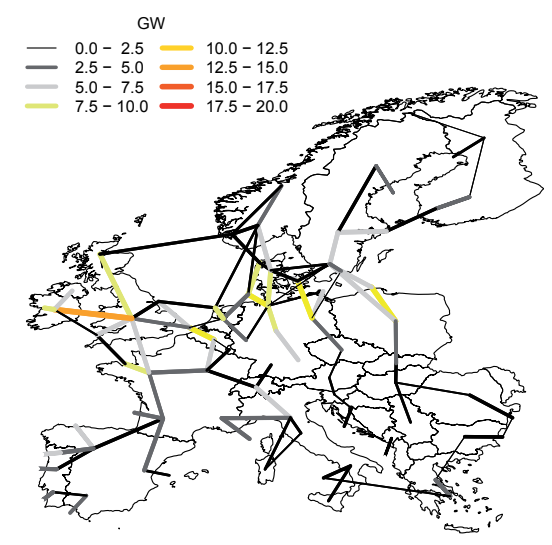

$\alpha=30 \%, \beta=60 \%$ to $100 \%$

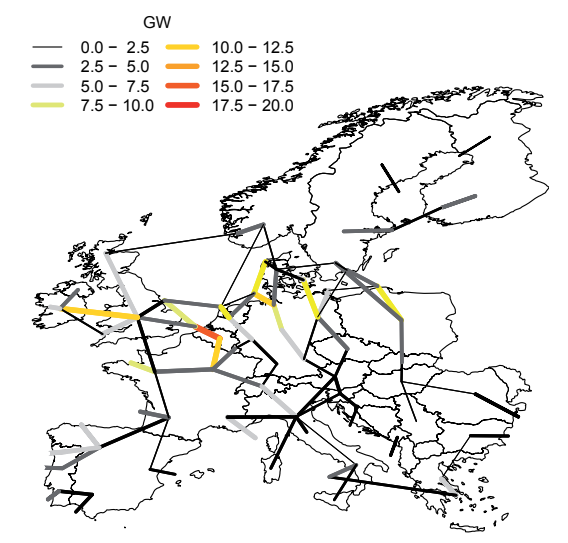

Figure 6: "Must have" grid. Minimal grid extensions on top of existing ENTSO-E grid for varying wind share between $60 \%$ and $100 \%$.

\subsection{Mix of backup supply and emissions reduction}

The major grid extensions presented in the previous section facilitate the integration of VREs to the power system considerably, but do not solve all problems. Dispatchable power plants still have to adopt to the fluctuating supply of VREs. In our model URBS-EU, power plant dispatch is optimized as well and we can therefore analyse the suitability of different backup technologies for each VRE configuration: Increasing flexibility is needed with VRE penetration rise.

Figure 7 shows the contribution of different power plant types to the satisfaction of residual load and the resulting emission reductions. As the backup energy mix strongly depends on the installed capacity of thermal power plants, we analyse two scenarios. The left column of Figure 7 shows the backup 
energy mix for a scenario, where today's capacity mix is assumed. The right column shows the backup energy mix for a cost-optimal capacity mix for each VRE scenario.

With increasing VRE share, more flexible backup technologies are necessary. Coal and nuclear, today's rather inflexible mid- and baseload power plants, become unsuitable with increasing VRE shares. In turn, more flexible technologies, such as gas, hydro and biomass power plants, are needed. The extent of this fuel switch however depends on the speed of VRE capacity build up. If Europe realized one of the VRE scenarios instantaneously, today's backup capacities would still be on line and the left column would be valid. If the build up of VRE capacity takes longer than the lifetime of the existing power plants, the right column is closer to reality.

While existing coal and nuclear power plants can still supply backup up to larger shares of VRE, the reduction of FLHs for coal and nuclear power plants through VRE penetration renders new investments in these capital-intensive technologies uneconomic in terms of overall costs. This will be the case for VRE penetration above $30 \%$. A remarkably sharp threshhold occurs, as can be seen in the right column of Figure 7 .

As both scenarios contain cost-optimal grid extensions, the thresholds for technology suitability have to be understood as upper limits. Especially for regions with high VRE shares inflexible backup technologies face economic difficulties at lower overall $\alpha$ if fewer grid extensions are realized (Schaber et al., 2011).

The last row of Figure 7 shows the emission reduction for both scenarios. It increases with $\alpha$ and is determined by coal phase-out and the role of gas for 
higher VRE shares.

Using existing backup Cost optimal backup ca-

capacities
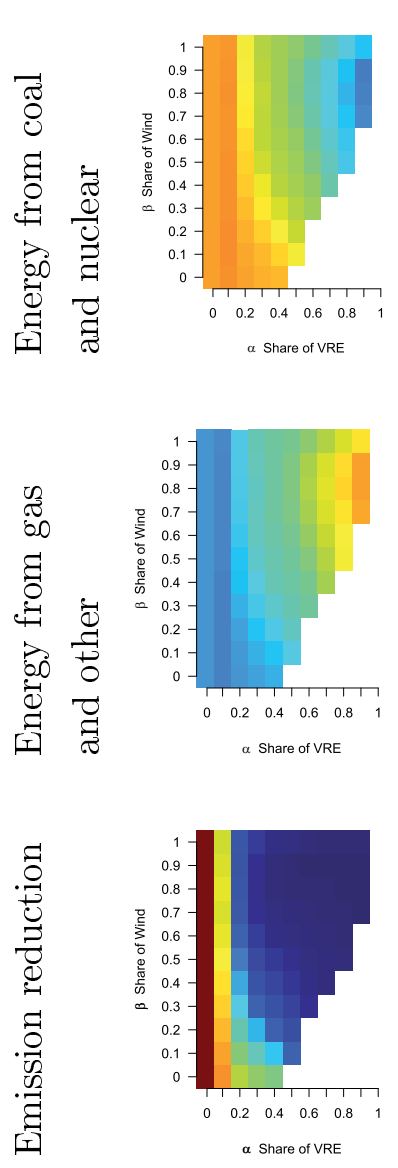

pacities
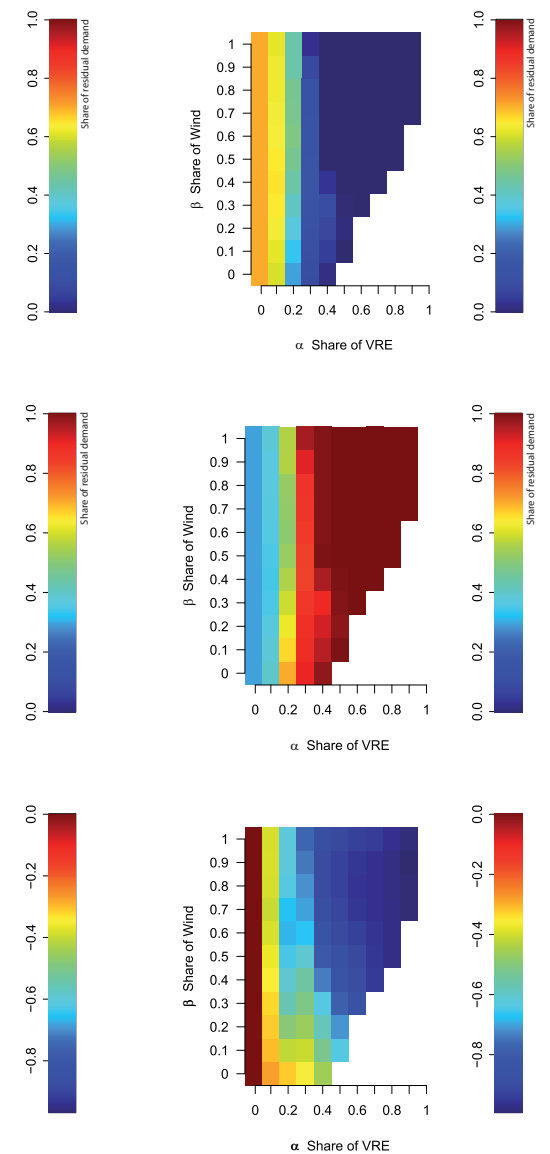

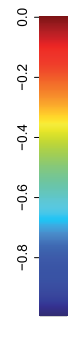

$\alpha$ Share of VRE

Figure 7: Energy by conventional power plants and emission reduction. The backup energy is shown as share of total residual demand, beeing total load to be supplied by backup power plants. "Other" stands for bioenergy, hydro and oil power plants.

\subsection{Casestudy: $60 \%$ VRE penetration in 2050}

We studied a large range of VRE scenarios and presented their respective impacts on the power system. To provide guidance to the reader, we apply 


\begin{tabular}{l||ll} 
& Optimal Grid & No Grid \\
\hline \hline Ideal mix to achieve & $\beta=85 \%$ & $\beta=65 \%$ \\
minimal $\Delta$ at $\alpha=60 \%$ & & \\
\hline Parameter values at the identified point & \\
\hline Misfit $\Delta$ & $40 \%$ of total demand & $50 \%$ of total demand \\
Overproduction & $<1 \%$ of total demand & $10 \%$ of total demand \\
Backup capacity & $80 \%$ of peak load & $100 \%$ of peak load \\
CoE (high VRE costs) & $140 € /$ MWh & $150 € /$ MWh \\
CoE (low VRE costs) & $80 € /$ MWh & $90 € /$ MWh \\
Grid integration costs & $3 € / M W h$ (load) & -
\end{tabular}

our results to an exemplary VRE scenario and sketch a possible roadmap how to reach this scenario from today's situation.

We define our exemplary scenario based on the following assumptions. Until 2050, strong emission reductions have to be achieved by the European power sector. Wind and solar energy will play an important role in the electricity supply in 2050 and satisfy $60 \%$ of electricity load. Based on our results, advantageous design features of a power system with $60 \%$ VRE penetration can be determined.

Table 2: Power system properties with VRE penetration of $60 \%$.

Figure 3 provides a good overview on the integration challenges of $60 \%$ VRE penetration and the role of grid extensions. With minimal misfit $\Delta$, lowest adaption needs of the power system occur, as supply is closest to demand. As can be read from the last row of Figure 3, minimal misfit $\Delta$ for a VRE share of $60 \%$ translates to wind share of $85 \%$ with and to $65 \%$ 
without grid extension (see Table 2). The absolute misfit is lower with grid. Without grid extensions, wind supply is not smooth enough and thus does not fit very well to the demand. The systematic advantage of solar energy for low connection cases due its good fit to the diurnal load pattern re-emerges. Table 2 also shows that through grid extension, the necessary backup can be reduced by $20 \%$, i.e., around 100 GW. Overproduction amounts to $10 \%$ without grid and less then $1 \%$ with grid extension. About $300 \mathrm{TWh}$ excess production can thus be avoided with grid extensions. Also, the CoE, driven by total - backup and VRE - capacity can be lowered by $10 € / M W h$, while the costs for the grid only amount to $3 € / M W h$. Thus, the transmission grid extensions, coming at total investment costs of 150 billion $€$, are costeffective. Until 2050, large parts of today's power plants will be shut down and an adapted backup capacity mix is possible. Following our analysis in Figure 17, only flexible power plants, mainly gas, will be suitable for a VRE penetration of $60 \%$. The achieved emission reduction through the VRE capacity is roughly $85 \%$ (Figure 7) in the case with grid extension. Due to the fuel switch to gas power plants, a more flexible technology, the emission reduction is larger than the share of VRE.

An advantageous system with $60 \%$ VRE should thus have a high share of wind energy, ideally $80 \%$ and a powerful transmission grid to integrate wind and solar energy. It should also provide backup energy from flexible power plants, such as biogas, hydro or gas power plants.

Today's VRE penetration in Europe amounts to $5 \%$ with a contribution of wind of $86 \%$ (IEA, 2011b). The necessary steps in terms of system design to reach $60 \%$ VRE penetration with more than $60 \%$ wind in 2050 can be 
487 identified from our results. A total of about $1500 \mathrm{GW}$ wind and solar capacity ${ }_{488}$ has to be built until 2050, i.e., $38 \mathrm{GW}$ /year in Europe. In 2009 and 2010, each ${ }_{489}$ year the last two years, $18.5 \mathrm{GW}$ of new wind and solar PV capacities have 490 been installed on average (GWEC, 2011; EPIA, 2011). The build up speed 491 thus has to be doubled. Second, transmission grid extensions should be built 492 to integrate these large capacities. The necessary grid capacity extensions ${ }_{493}$ amount to about the triple of the existing grid and planning should therefore 494 start as soon as possible. Important grid corridors are shown in Figure 6. ${ }_{495}$ Finally, new dispatchable capacities, which will serve as backup for the VREs, 496 should be chosen in accordance to the increasing flexibility requirement. 


\section{Discussion and Conclusion}

In this paper, we analyze the integration challenges of VREs in Europe and the role of grid extensions as a measure to alleviate these. We apply the model URBS-EU, an advanced model of the European power system, to analyze each possible VRE configuration on the defined parameter space, spanned by the VRE penetration $\alpha$ and the mix between wind and solar PV energy $\beta$. This parametric study allows us to identify advantageous system designs features for each level of VRE penetration, insights of high relevance for the conception of renewable energy policies and long term targets. We find that generally, major integration challenges occur with increasing VRE penetration: Large backup capacity is required and important overproduction and misfit between supply and demand occur. Grid extensions alleviate these challenges, as they smoothen the temporal fluctuations and remedy the geographical dispersion of VRE. As a consequence, required backup capacity, overproduction and the misfit between VRE supply and demand are reduced. Grid integration costs remain below $25 \%$ of the VRE investment costs for all concievable VRE scenario. From our results the most important corridors for new transmission lines can be identified. Finally, we show that increasingly more flexible power plants are needed with rising VRE penetration.

Based on eight-year meteorological timeseries of wind and solar availability, it is proven, that VRE penetration of more than $80 \%$ is possible. These high VRE shares, however come at the cost of large wind and solar capacities, excelling the peak load by up to 10 times. Due to the restricted availability of VRE with annual utilization rates of $30 \%$ or lower (i.e., FLHs of 2600 or 
lower), a VRE powered system always needs larger total capacity, than a system with dispatchable power plants. On top of that, large backup capacities are needed to ensure reliable power supply. These large capacities lead to high CoE generation of up to $130 € / \mathrm{MWh}$ in the most extreme cases (low costs case). With grid extensions, the necessary backup and VRE capacities can be reduced and with it the costs of producing electricity. With an optimal CoE of roughly $80 € / M W h$ result. These are below the CoE of coal power plants of $115 € / M W h$, if a high carbon costs of $100 € / t$ is assumed. Furthermore, the overproduction and the misfit between supply and demand are lowered through grid extensions. This entails less adaption need for the remaining power system, being backup power plants and storage.

The mix between wind and solar energy also influences the above discussed system properties. Firstly, solar PV cannot provide power at night and thus, VRE shares above $40 \%$ can not be realized with solar energy only. To reach higher VRE shares, an important contribution of wind energy $40 \%$ or more - is necessary. Wind energy can - especially in combination with grid extensions - provide a "fairly smooth" supply. For large $\alpha(>40 \%)$ this furthermore results in lower backup capacity requirements and misfit between supply. Wind is necessary for high VRE penetration and has further important advantages compared to solar energy, especially in combination with grid extensions. Without grid extensions, wind supply is less smooth and does not fit very well to the demand, while the diurnal pattern of solar energy fits rather well to the diurnal load pattern. Thus, without grid extensions, a larger share of solar energy is favorable to have important shares of solar energy, around $30-50 \%$ in the system.. 
Having identified the advantages of a powerful overlay grid, for the integration of VRE in Europe, the costs of such a "super-grid" are of high relevance. We quantified the grid integration costs for all possible VRE scenarios and find that the costs for such a grid reaches at most $6 € /$ MWh load for all VRE scenarios. This is roughly the double of the tariffs paid today in Germany, for example (ENTSO-E, 2011a). Grid integration costs generally increase with VRE share, but for very high shares, saturation effects occur. Solar energy needs less grid extension. This is due to the long correlation length of the solar availability pattern: It is night at about the same time all over Europe (see also Grotz, 2009) and therefore solar energy profits less from grid extension, than wind energy.

Generally, the additional grid capacities to be build are large. For the scenario investigated in our casestudy, the triple of today's grid capacity would be necessary. As the acceptance and building process for new transmission lines can reach up to ten years, early planning is crucial for the integration of VREs in Europe. We identified important connections in Europe for the integration of VREs. Those are strong north-south connections from Northern Scandinavia and Denmark to the load centers in Germany and France traversing the BeNeLux states and an offshore grid in the North Sea. Better connection of Spain and France and the connection of Italy over the Alps to the rest of Europe are crucial as well. To enable large shares of renewable energies, these transmission grid extensions should be on the EU's priority agenda for the next years.

The model only accounts for a macroscopic interregional energy transmission network, a European overlay network. The computations do not include net- 
work costs for upgrading the distribution networks, important especially for PV.

Apart from a powerful grid, future power systems with important VRE penetration need flexible backup power plants. Through increased interlinkage, VRE generation can be smoothened to some extent, but the high variability of the residual load remains. For investment in new power plants, the contribution of VRE to the future power supply should be taken into account. Our results show that for coal and especially for nuclear power plants a clear limit in suitability occurs: at VRE shares above $30 \%$, these power plants are uneconomic from a total system cost perspective, but flexible technolgies such as gas power plants are adequate.

Our analysis does not investigate storage as an integration option. We acknowledge its importance for VRE integration and recommend further research on the interaction of grid and storage, possibly based on these results. A related topic, which is not treated in this study, is the very short term variability of supply and ramping requirements for backup power plants. A closer look into the role and value of flexibility appears very promising. We furthermore recommend the application of a comparable methodology to other regions of the world to gain more general understanding of the drivers of grid extensions identified here.

The above described results of our parametric study can provide a helpful overview for the design of renewable energy policies. In a casestudy for VRE penetration of $60 \%$ in 2050 , we illustrate that our results can guide decisions about how to design a power system that is well adapted to the desired VRE share and thus faces minimal integration challenges. Transmission grid 
extensions are cost-effective and allow to reduce the required backup power by $100 \mathrm{GW}$ and overproduction by 300 TWh. To realize this target, political support is crucial: for capacity build-up of VREs and for planning and investment in grid extensions on the crucial corridors, that we identified.

01 VRE integration in Europe is very challenging and detailed information about the implications of high wind and/or solar shares in the power supply are vital for investment decisions, the conception of policies and VRE integration measures. We provide an overview of the system implication of all possible VRE scenarios in Europe and show, that a powerful overlay transmission grid reduces the adaption need of the remaining power system 67 considerably, while its costs remain below $6 € / M W h$ for all VRE configurations. 
${ }_{609}$ Appendix A. Model setup 


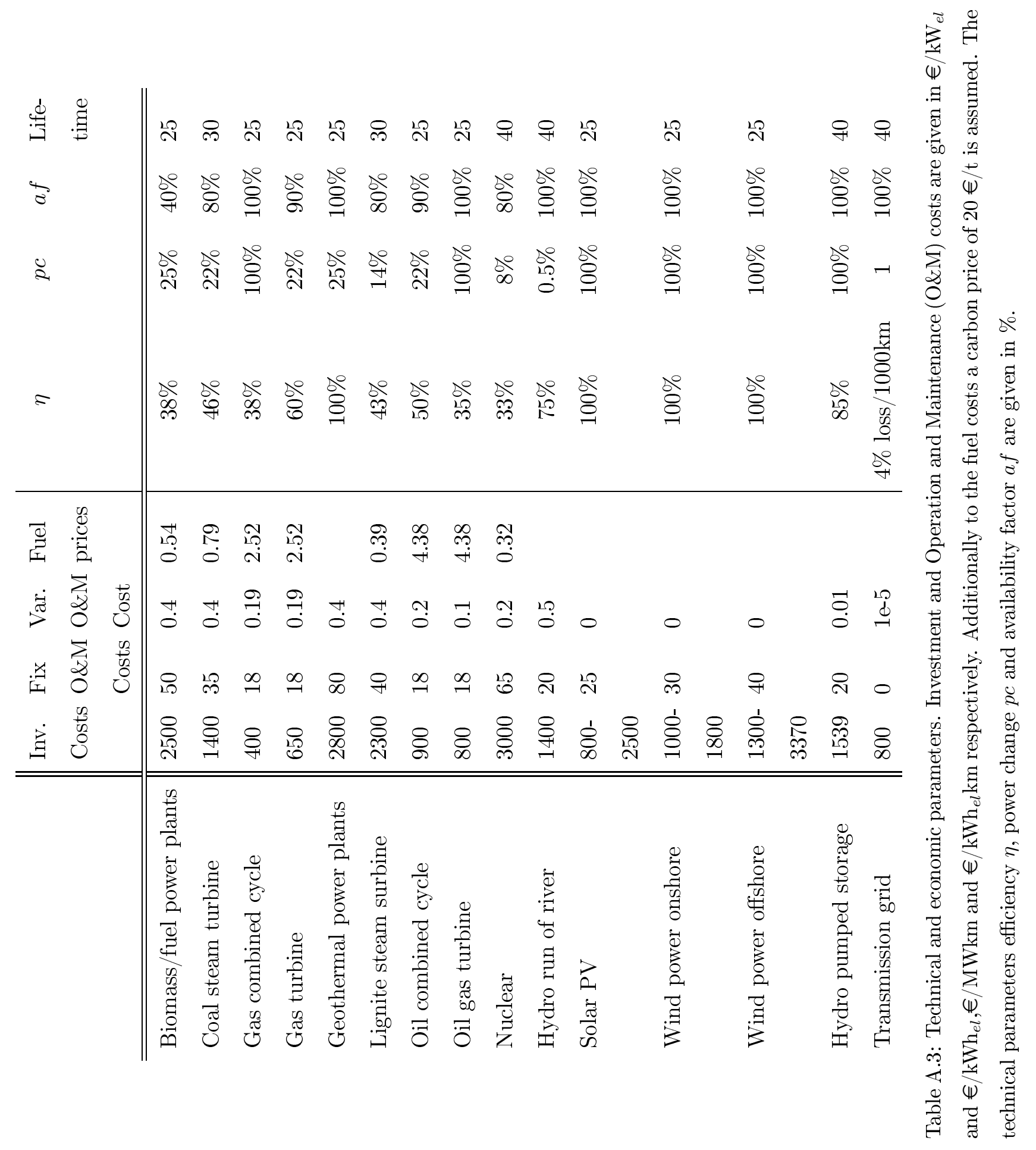


Aboumahboub, T., Schaber, K., Tzscheutschler, P., Hamacher, T. (Eds.), 2009. Optimization of the Utilization of Renewable Energy Sources in the Electricity Sector: accepted.

Biberacher, M., 2004. Modeling and optimization of future energy system using spacial and temporal methods. Ph.D. thesis, Institute of Physics, University of Augsburg.

Brückl, O., 2005. Globales potenzial der stromerzeugung aus windenergie: Endbericht der studie für die bmw ag. Ph.D. thesis, Technische Universität München, München.

Buckminster Fuller, R., 1971. The World Game: Integrative Resource Utilization Plannung Tool. World Resources Inventory.

Czisch, G., 2005. Szenarien zur zukünftigen Stromversorgung , Kostenoptimierte Variationen zur Versorgung Europas und seiner Nachbarn mit Strom aus erneuerbaren Energien (Scenarios for a future power supply, cost optimal scenarios for renewable power supply in Europe and its neighbors). Ph.D. thesis, Elektrotechnik / Informatik der Universität Kassel.

DLR, 2006. Trans-Mediterranean Interconnection for Concentrating Solar Power (TRANS-CSP), Final Report, Study by German Aerospace Center (DLR), Institute of Technical Thermodynamics, Section Systems Analysis and Technology Assessment and the Federal Ministry for the Environment, Nature Conservation and Nuclear Safety, Germany.

URL http://www.dlr.de/tt/desktopdefault.aspx/tabid-2885/

4422_read-6588/ 
Edenhofer, O., Knopf, B., Barker, T., Baumstark, L., Bellevrat, E., Chateau, B., Criqui, P., Isaac, M., Kitous, A., Kypreos, S., Leimbach, M., Lessmann, K., Magné, B., Scrieciu, S., Turton, H., Vuuren, D. v., 2010. The Economics of Low Stabilization: Model Comparison of Mitigation Strategies and Costs. Energy Journal 31.

ENTSO-E, 2010a. European network of transmission system operators for electricity: Load and consumption: Specifities for member countries. Brussels.

URL https://www.entsoe.eu/

ENTSO-E, 2010b. European Network of Transmission System Operators for Electricity: Statistical Data.

URL https://www.entsoe.eu/

ENTSO-E, 2011a. European Network of Transmission System Operators for Electricity: ENTSO-E Overview of transmission tariffs in Europe: Synthesis 2011.

URL https://www.entsoe.eu/market/transmission-tariffs/

ENTSO-E, 2011b. European Network of Transmission System Operators for Electricity: Net Transfer Capacities.

URL https://www.entsoe.eu/

EPIA (Ed.), 2011. European Photovoltaic Industry Association: Global Market Outlook for Photovoltaics until 2015. EPIA.

EWEA, 2010. European Wind Energy Association: Powering Europe: wind energy and the electricity grid. EWITS. 
Giebel, G., 2000. On the Benefits of Distributed Generation of Wind Energy in Europe. Ph.D. thesis, Fachbereich Physik der Universität Oldenburg.

Greenpeace, 3E, 2008. A North Sea electricity grid [r] evolution: electricity output of interconnected offshore wind power: a vision of offshore wind power integration.

URL http://www.greenpeace.org/raw/content/eu-unit/ press-centre/reports/A-North-Sea-electricity-grid-(r)evolution . $\operatorname{pdf}$

Grotz, B., 2009. Untersuchung der Korrelation zwischen Wind- und Solarangebot mit spezeiller Berücksichtigung von Extremwetterlagen, Diplomarbeit. Ph.D. thesis, Universität Augsburg.

GWEC (Ed.), 2011. Global Wind Energy Council: Global Wind Report; Annual Market Update 2010. GWEC.

Heide, D., von Bremen, L., Greiner, M., Hoffmann, C., Speckmann, M., Bofinger, S., 2010. Seasonal optimal mix of wind and solar power in a future, highly renewable europe. Renewable Energy 35 (11), 2483 - 2489.

Hoogwijk, M., 2004. On the global and regional potential of renewable energy sources. Ph.D. thesis, Universiteit Utrecht.

IEA, 2010a. International Energy Agency: Interactive Renewable Energy Calculator: RECaBS.

URL http://www.recabs.org/

IEA, 2010b. International Energy Agency: World Energy Outlook 2010. OECD, Paris. 
IEA, 2011a. International Energy Agency: Deploying Renewables: Best and Future Policy Practice. OECD, Paris.

IEA, 2011b. International Energy Agency: World Energy Outlook 2011. OECD, Paris.

IPCC, 2011. IPCC Special Report on Renewable Energy Sources and Climate Change Mitigation. Tech. rep.

Kuhn, P., Kühne, M., 2011. Optimierung des Kraftwerks- und Speicherausbaus mit einem interativen und hybriden Modell. VDI-Bericht 2157, 305317.

McKinsey, KEMA, The Energy Futures Lab at Imperial College London, Oxford Economics, European Climate Foundation, 2010. RoadMap 2050: A practical guide to a prosperous, low-carbon Europe: Technical Analysis: Volume I.

URL http://www.roadmap2050.eu/

Pieper, C., Rubel, H., 2011. Revisiting Storage. There Is a Business Case. Boston Consulting Group.

PWC, PriceWaterHouseCoopers, Potsdam Institute for Climate Impact Research, International Institute for Applied System Analysis, European Climate Forum, 2010. 100\% Renewable Energy: A Roadmap to 2050 for Europe and North Africa.

Schaber, K., Steinke, F., Hamacher, T., 2011. Transmission Grid Extensions for the Integration of Variable Renewable Energies: Who Benefits Where? Energy Policy Submitted. 
TradeWind, 2009. Integrating Wind - TradeWind: Developing Europe's 703 power market for the large-scale integration of wind power, Study by Sintef ${ }_{704}$ Energieforskning AS, Riso DTU, 3E, Kema Nederland BV, Technical 705 Research Centre of Finland, Garrad Hassan and Partner Ltd, Tracabel 706 Engineering and Deutsche Energie-Agentur GmbH (dena). URL http://www.ewea.org/fileadmin/ewea_documents/documents/ publications/reports/TradeWind_Report_01.pdf

Tzscheutschler, P., 2005. Globales technisches potenzial solarthermischer stromerzeugung. Ph.D. thesis, Lehrstuhl für Energiewirtschaft und An711 wendungstechnik, Technische Universität München. 